\title{
Conformational analysis of chiral polymers in solution
}

\author{
Francesco Ciardelli and Piero Salvadori \\ Department of Chemistry and Industrial Chemistry, University of Pisa \\ CNR Centro Macromolecole Stereoordinate e Otticamente Attive,56100 Pisa,Italy
}

\begin{abstract}
Relationships between chiroptical properties (Optical Rotatory Dispersion, ORD, and Circular Dichroism, CD) and Conformation of structurally simple isotactic polymers and copolymers derived from optically active monomers are reviewed. Both experimental and calculated values fit with chain conformations consisting predominantly of one screw sense helical sections due to dissymmetric cooperative interactions among chiral side chains. This effect can be transmitted along isotactic sequences of units derived from a nonchiral comonomer, which than contribute to copolymer ORD and $\mathrm{CD}$. In particular this latter shows a typical "couplet" connected to exciton splitting due to interactions among chromophoric moieties disposed in a mutual chiral arrangement with a predominant handness. Similar phenomena are observed also in the corresponding nonstereoregular polymers even if to a lower extent, thus suggesting the existence of local order determined by next-neighbour interactions.
\end{abstract}

\section{INTRODUCTION}

While in case of biopolymers a clear distinction between secondary and tertiary structure was made since the early times, conformational problems in synthetic polymers were mostly devoted to the investigation of the tertiary structure for a better understanding of their unperturbed dimensions, with much less attention given to the local spatial arrangement of the atoms. Nowdays the ever increasing interest for the use of synthetic polymers in very special sophisticated applications (Speciality polymers) makes the problem of local conformation very up to date. Indeed the application as catalysts, chemical reagents, drugs, liquid crystals and so on are more dependent, given a certain primary structure, on secondary rather than tertiary structure of the macromolecules involved. Moreover many of this applications are carried out in solution and optical activity can provide a very convenient tool for providing information on the conformation at the local molecular level, being sensitive to even modest geometrical changes in the molecule.

Indeed in case of biopolymers these techniques have supplied fundamental contribution when specific ordered structures exist (1) and valid theoretical approaches are available for calculating chiroptical properties, rotatory dispersion (ORD) and circular dichroism (CD), to be compared with experimental results (2).

In spite of the large number of optically active synthetic polymers prepared in the last 25 years, only few examples exist in which a definite relationship between chiroptical properties and conformation has been established. However these last are really useful for a better understanding of the structural properties of. synthetic polymers in solution; these are.very important for the above mentioned special applications and can be also often extrapolated successfully to the more complex biological polymers $(2,3)$.

ISOTACTIC POLYMERS OF OPTICALLY ACTIVE 1-OLEFINS

Title polymers provided an unique example as the paraffinic backbone of their chains allows a simple and reliable conformational analysis and molecular structure in the crystalline state is known $(4,5)$ with a high level of accuracy. Also, the simple chemical structure implies the existence of electronic transitions concerning only carbon and hydrogen in a paraffinic array and than located below $180 \mathrm{~nm}$ and not very different in energy. 
As this last spectral region was far below the limit of commercial CD instruments, the original studies were performed by measuring rotatory power at a wavelength higher than the electronic transition by ORD techniques. Very recently-it has been finally possible to measure even $C D$ curves by vacuum UV dichrograph, as discussed ahead.

Going back to the fundamental results obtained in the $60(6)$, it is necessary to consider firgtly that the isotactic polymers derived from one enantiomer of chiral 1-olefins, with an asymmetric carbon atom in 1 or 2 position to the double bond, showed rotatory power with an absolute value remarkably higher than low molecular weigth structural analogs, that is strictly resembling the primary chemical structure of the repeating unit. On the other side the rotatory power of the above polymers was very close to that of low molecular weight "conformational models" where the conformational situation, the secondary structure, is the same as for the repeating unit in the isotactic macromolecules. These data are summarized in Table 1 and represented in Figure 1 for the simple chiral olefin, 4-methyl-1-hexene (6).

TABLE 1

Conformational Features and Optical Rotatory Power of Isotactic Poly-(S)-4-methy1-1-hexene and Low Molecular Weight "Structural" an "Conformational" Models

\begin{tabular}{|c|c|c|c|c|}
\hline & $\begin{array}{l}\text { Number of } \\
\text { Alloved } \\
\text { Conformations }\end{array}$ & \multicolumn{2}{|c|}{$\begin{array}{c}\text { Optical Rotatory Power } \\
{[\Phi]_{\mathrm{D}}}\end{array}$} & $\operatorname{Ref}$. \\
\hline $\begin{array}{l}\text { Isotactic Poly-(S)-4 } \\
\text {-methy 1-1-hexene }\end{array}$ & $2^{a)}$ & $+288^{b)}$ & $+240^{a, b,)}$ & 6 \\
\hline $\begin{array}{l}\text { Structural mode1 } \\
{[(\mathrm{S})-2,4-\text { dimethyl-hexan }}\end{array}$ & e] & +22 & +20 & 6 \\
\hline $\begin{array}{l}\text { Conform tional mode } 1 \\
{[(2 \mathrm{R}, 5 \mathrm{~S})-2,2,4,5 \text {-tetra }} \\
\text { methyl-hexane }]\end{array}$ & - & +120 & +120 & 7 \\
\hline
\end{tabular}

a) For the favored left-handed helix b) Based on one repeating unit

Poly-(S)-4-methyl-1-hexene, isotactic lefthanded helix<smiles>CC[C@H](C)C[C@H](C)CC</smiles><smiles>CC[C@@H](C)C[C@H](C)CC</smiles>

(s)-2,4-dimethyl-hexane, structural model<smiles>CC[C@H](C)CC(C)C</smiles><smiles>CC[C@@H](C)CC(C)C</smiles><smiles>CC[C@H](C)CC(C)C</smiles>

(3R,5S)-3,3,3,5-tetramethyl-heptane, conformational model<smiles>CC[C@H](C)CC(C)C</smiles><smiles>CC[C@@H](C)CC(C)C</smiles>

Fig. 1. Allowed Conformations of the Repeating Unit of Isotactic Poly-(S)-4-methyl-1-hexene and of Its Monomeric Models. 
It is important to point out that the few conformations allowed (the ones with the lowest internal energy) for the "conformational mode1" (7) correspond to those of the repeating unit when assuming for the isotactic macromolecule an one screw sense helical chain conformation, the selected screw sense corresponding to that experimentally observed in the crystalline state for the same monomer enantiomer (8). The validity of the assumption, that the predominant screw sense foreseen on the basis of "a priori" conformational analysis(6) and experimentally observed by $\mathrm{X}$-ray is the same in solution, was nicely confirmed by measuring the rotatory power in solid state which resulted practically the same as in the solution (9), at least when the macromolecules assume in the lattice helical conformations of one single handness.

This result brought to the hypothesis that isotactic polymer chain sections of the above chiral olefins maintained in solution one screw sense helical secondary structure similar to that present in the crystalline state, which allows to minimize intramolecular steric interactions. Clearly the conformation in solution is characterized by helical blocks with shorter average length than in the crystals and connected through conformational reversals $(8,10)$. Thus: It can be considered as consisting of a succession of chain sections spiralled in both screw senses; the average length of the sections in the favored screw sense markedly prevailingly. Further support to such hypothesis derived from the calculation of the rotatori power of low molecular weight models and polymers; in case of these last agreement between experiments and calculations was indeed observed only when assuming the predominant screw sense for the isotactic chains(6) (Table 1 ). The driving force responsible for the prevalence of a single screw sense was proposed to be the cooperative interaction among chiral side chains disposed along the isotactic backbone $(8,11)$. According1y the rotatory power absolute

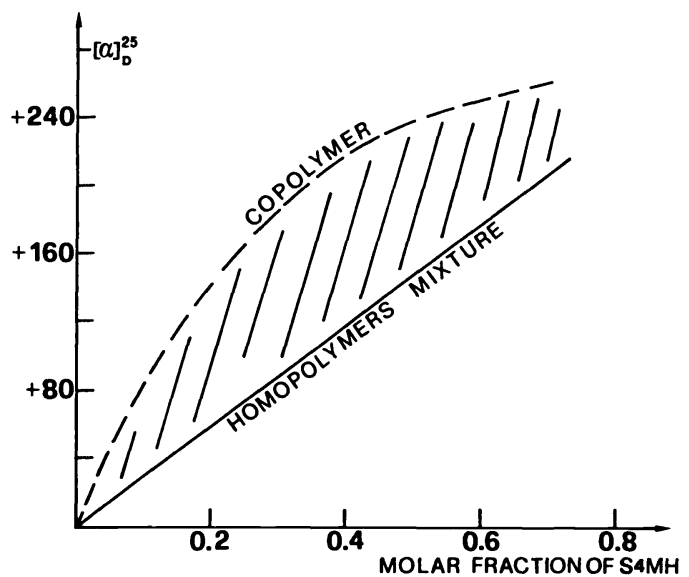

Fig. 2. Rotatory Power vs. Composition of Coisotactic (S)-4-Methy 1-1-hexene (S4MH) /4-Methy1-1-pentene Copolymers and Isotactic Homopolymers Mixtures.

value decreases when going to less stereoregular fractions $(6,8)$ and by random insertion of ethylene units in the poly-1-olefin chain (11); both reducing the average length of isotactic blocks.

Interestingly, the above cooperative effect is maintained by random insertion of units deriving from a nonchiral l-olefin, in the isotactic chain derived from the chiral 1-olefin, the effect being more evident if the former has similar conformational properties as the chiral comonomer. Accordingly coisotactic random copolymers of (S)-4-methy1-1-hexene (S4MH) with 4-methyl-1-pentene (4MP), which give isomorphous repeating units, show rotatory power markedly higher than the corresponding homopolymers mixtures indicating that $4 \mathrm{MP}$ units in the copolymer have optical activity of the same sign as S4MH units (Figure 2).

The cross-hatched area within diagonal and curve in Figure 2 corresponds to the integrated rotatory power of 4MP units in the copolymer in the whole range of composition.

This result can be interpreted in the frame of the proposed conformational model by assuming that the copolymer rotatory power per average monomeric unit is given by the following equation:

$$
\mid \Phi]_{c}=N_{\mathbf{h}}\left[\left.\Phi\right|_{\mathbf{h}}+N_{\mathbf{p}}[\Phi]_{\mathbf{p}}\right.
$$

where $\mathrm{N}$ indicates the molar fraction of units derived from S4MH and 4MP, respectively, $[\boldsymbol{\Phi}]_{\mathbf{h}}$ is the molar rotatory power of one $\mathrm{S} 4 \mathrm{MH}$ unit in the isotactic chain and $\mid \Phi]_{p}$ is the molar rotatory power of one 4MP unit in the copolymer. 
Thus it is possible to calculate $[\phi]_{\mathbf{p}}$, that is the rotatory power of one $4 \mathrm{MP}$ unit in the copolymer macromolecules; this value increases with increasing tacticity degree and the content of S4MH units approaching the value of +240 calculated for one 4MP unit inserted in a lefthanded helical chain which is the favored one when the chiral side chain have (S) absolute configuration as in the present case (12). It is noteworthy that $[\Phi]$ p practically reaches an asymptotic value already in the copolymer containing equimolar amounts of the two olefins. This evidently indicates that even short sections of 4MP units assume the secondary structure corresponding to one screw sense helicity of the main chain under the inductive effect of the chiral comonomer units. From the rotatory power, as compared with the theoretical limiting value of 240 , it is possible to evaluate for the most isotactic polymer fraction with $50 \%$ or less 4MP units that more than $80 \%$ of these last are included in helical sections spiralled in the favored screw sense. Such value fits very well with the value of $92 \%$ calculated by statistical approaches for isotactic poly-(S)-4-methyl-1-hexene (13). In conclusion it is possible to describe the conformation of the coisotactic 4MP/S4MH copolymer with a left-handed $7 / 2$ helix (the conformation of isotactic homopolymer of (S4MH) where a fraction of sec.buty.1 groups have been replaced by isopropyl groups (Fig. 3).

The pure conformational origin of the peculiar optical rotatory properties of the isotactic macromolecules derived from chiral 1-olefins was in keeping with ORD measurements which followed strictly a one term Drude equation:

$$
[\boldsymbol{\Phi}]_{\hat{\lambda}}=K\left(\lambda^{2}-\lambda_{0}^{2}\right)
$$

with $\lambda_{0}$ substantilly the same in polymers and low molecular weight models, thus soggesting that the electronic transitions responsible for the chiroptical properties were the same in the two classes of compounds, different absolute values of the optical rotation being due to different values of $\mathrm{K}(6 ; 8)$.

An experimental confirmation of this explanation was supplied recently by vacuum UV CD measurements on films of poly-(S)-4-methyl-1-hexene (PS4MH) and poly-(R)-3,7-dimeth1-1-octene (PR37 DMO). Both polymers samples showed a dichroic band cendered at $158 \mathrm{~nm}$ which allowed to calculate ORD curves in keeping with the experimental ones by Kronig-Kramers transforms thus indicating that this dichroic band is responsible for the observed chiroptical properties (14).

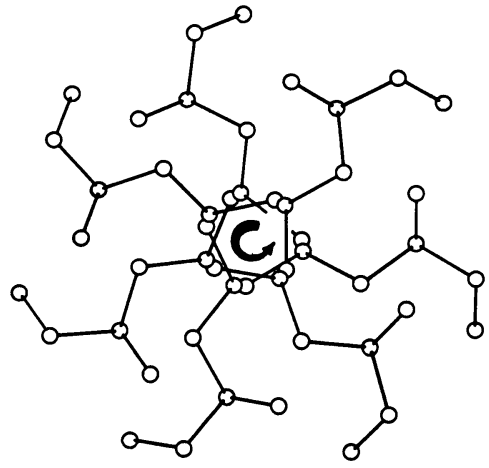

POLY-(S)-4-METHYL-1-HEXENE

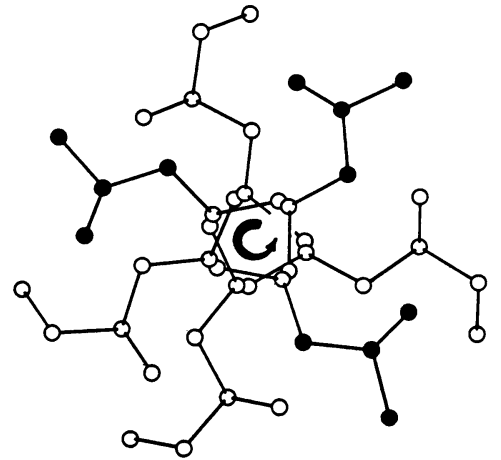

(S)-4-METHYL-1-HEXENE / 4-METHYL-1-PENTENE COPOLYMER

Fig. 3. End view of a left-handed 72 helix of isotactic poly-(S)-4-methyl-1-hexene and of coisotactic (S)-4-methy1-1-hexene/4-methy1-1-pentene copolymers.

Taking into account the proposed conformational model it was predicted by several authors that optically active isotactic poly-l-olefins should display dimensions largely differing from those of optically inactive polymeric analogs. This prediction and then an additional proof in favor of the proposed model, was indeed obtained by estimating from viscosity data unperturbed dimensions of isotactic optically active PS4MH and isotactic P4MP which is not optically active. The characteristic ratio $\bar{h}_{0}^{2} / \mathrm{nl}^{2}$ for the optically active polymer was distinctly greater than that of optically inactive P4MP as shown in table 2 (15). 
TABLE 2

Observed and Calculated Characteristic Ratios of Isotactic Polymers from Optically Active and Nonoptically Active 1-0lefins (After Ref. 15)

\begin{tabular}{lcccr}
\hline & \multicolumn{2}{c}{ CHARACTERISTIC RATIOS } & \multicolumn{2}{c}{ CALCULATED } \\
POLYMER & $\overline{\mathrm{h}}^{2}{ }^{2} / \mathrm{n}^{2}$ & $\mathrm{~T},{ }^{\circ} \mathrm{C}$ & $\overline{\mathrm{h}}^{2} 0^{/ \mathrm{nl}^{2}}$ & $\mathrm{~T},{ }^{\circ} \mathrm{C}$ \\
\hline POLY-(S)-4-METHYL-1-HEXENE & 11.2 & 133 & 13.2 & 27 \\
& 9.4 & 165 & 10.1 & 127 \\
POLY-4-METHYL-1-PENTENE & 8.1 & 233 & 7.8 & 227 \\
\hline
\end{tabular}

\section{ISOTACTIC POLYMERS WITH AROMATIC SIDE CHAINS}

Aromatic chromophores are very useful probes for the conformational analysis of macromolecules by chiroptical techniques. Indeed benzene is characterized by three absorption bands in the near UV connected to its $\pi$-electrons system ( $L_{a}, L_{b}$ and $B$ in order of increasing energy). In coisotactic copolymers of optically active 1-olefins with viny1 aromatic monomers, such as styrene and vinyl naphthalenes, the only absorbing moiety over $180 \mathrm{~nm}$ is the aromatic chromophore itself and no others electronic transitions are observed in the accessible spectral region (16). Accordingly, while in ORD spectra a background optical rotation from high energy transitions, such as the $158 \mathrm{~nm}$ dichroic band in polyolefins (17) exists, the CD speçtra over $180 \mathrm{~nm}$ can show optically active bands due only to the $\pi$-electrons of the aromatic ring. Thus coisotactic random copolymers of styrene with R37DMO show dichroic bands in the 260-180nm spectral region which must be attributed to the phenyl chromophore and supply an evident proof that styrene units are in a chiral arrangement with a predominant handness. In these copolymers (17) all the three mentioned electronic transitions occur in an optically active environment and generate rather strong ellipticity, higher in absolute value than for low molecular weight structural models for which, due to the unfavorable dissymmetry factors, only the lowest energy transition has a detectable ellipticity. On the other side the conformational model (R)-2,2-dimethy1-3-phenyl-butane(18), where the phenyl group is practically in the same spatial situation as in the monomeric unit of isotactic right handed polystyrene, shows ellipticity of the same order of magnitude as the copolymer (Table 3). This result is in keeping with those obtained for homopolymers of aliphatic optically active olefins described in the previous chapter.

Moreover in the CD spectrum of styrene/37DMO (20/80) copolymer a couplet is osservable in the region of the $\mathrm{I}_{\mathrm{B}}$ electronic transition, whereas the conformational model shows only a single dichroic band even if of the same order of intensity (17). This last can be attributed to the dissymmetric perturbation of the single phenyl chromophore by the asymmetric carbon atom dire ctly bound to $i t$, whereas the couplet in the copolymer is due to the exciton coupling of ${ }_{B}$ electronic transition of aromatic groups chirally disposed along the macromolecular chain. An analogous behavior was observed in several other coisotactic random copolymers of a single enantiomer of 37DMO with additional vinyl aromatic monomers such as o-(17),p-methyl-styrene (19), 1-viny1-(20) and 2-viny1-naphthalene (21) and 6-vinylchrysene (22) (Table4). By contrast no couplet and a single chiral band only, as in the low molecular weight conformational model was detected in the analogous coisotactic copolymers of allylbenzene with R37DMO, where the phenyl ring is not directly bound to the main chain as in the previous cases, but spaced by one methylene group (23).

TABLE 3

Circular Dichroism of Coisotactic (R)-3,7-dimethyl-1-octene / Styrene (80/20) copolymer and Its Low Molecular Weight "Structural"and "Conformational" Models (After Ref. 16)

\begin{tabular}{|c|c|c|c|}
\hline \multirow[t]{2}{*}{ COMPOUND } & \multicolumn{3}{|c|}{ CIRCULAR DICHROISMa), $\Delta \varepsilon(\lambda)$} \\
\hline & ${ }^{1} L_{b}$ & $1_{L_{a}}$ & $1_{\mathrm{B}}$ \\
\hline COPOLYMER b) & $-0.24(262)$ & $-3.5(215)$ & $\begin{array}{l}-19.0(196) \\
+10.9(188)\end{array}$ \\
\hline $\begin{array}{l}\text { STRUCTURAL MODEL } \\
\quad(3 \mathrm{~S}, 9 \mathrm{~S})-3,9-\text { dimethy } 1- \\
-6-\text { pheny1 undecane }\end{array}$ & $+0.02(260)$ & n.d.c) & n.d.c) \\
\hline $\begin{array}{l}\text { CONFORMATIONAL MODEL } \\
\text { (R) -2-pheny1-3,3- } \\
\text {-dimethy1 butane }\end{array}$ & $-0.17(258)$ & $-3.0(209)$ & $-24.5(188)$ \\
\hline
\end{tabular}

a) In the phenyl chromophore absorption region. b) Per aromatic unit, $\lambda=n m$. c) Too low to be determined. 
A final confirmation of the onescrew sense helical conformation of these chiral polymers (Figure 4) was obtained by calculating (24) circular dichroism curve for given chain conformations performed on the basis of the De Voe model. This last is a coupled oscillators theory of molecular optical properties and then, the relative geometry of the interacting chromophores as well as the description of their polarizability have to be provided. In case of vinyl aromatic copolymers calculations were performed by assuming for the chromophores the same geometry as in the helical chain found for the corresponding homopolymers in the crystalline state with left or right handness depending on the (S) or (R) absolute configuration of the l-olefin enantiomer used as comonomer, respectively. Indeed according to previous deductions (S)-side chains induce a lefthanded helicity, whereas the right handness is generated by $(R)$-olefins(8). The aromatic chromophore, in the region of the electrically allowed " $1_{B}$ transition, was described by two ascillators, placed in the middle of the aromatic ring and directed as in the corresponding methylsubstituted aromatic hydrocarbons. For example in the case of benzene the two oscillators are directed along two mutually orthogonal directions, in the plane of the benzene ring itself. The imaginary part of polarizability for these oscillators was obtained from absorption spectra of low molecular weight aromatic hydrocarbons (a.e. toluene for styrene copolymers), while the real part was derived from it through Kronig-Kramers transform.

Calculated CD spectra resulted in a very good agreement with those detected experimentally for the coisotactic copolymers of one enantiomer of 37DMO with styrene, o-,p-methylstyrene, 1-and 2-vinylnaphthalene (Table 4). This proved further that the origin of the couplet in the region of the $1_{\mathrm{B}}$ electronic transition was the mutual electrostatic interaction of the transition moments of aromatic chomophores in the side chains disposed along the one screw sense helical secondary structure (16).

The same calculations allowed to get a convincing explanation for the presence of a single dichroic band, instead of a couplet, in the ${ }_{B}$ transition region in the CD spectrum of the allylbenzene copolymers. It was actually found that exciton dipole-dipole interactions between aromatic side chains disposed according to one of the two quasi equienergetic conformations of the repeating unit allowed by the methylene spacing group, even if inserted in an isotactic one screw sense chain, give opposite $\mathrm{CD}$ contributions (Fig. 4). As the consequence the only osservable $\mathrm{CD}$ is that due to the dissymmetric perturbation of a single phenyl group in the one screw sense helix and corresponds closely to that of the low molecular weight conformational model showing only one dichroic band (23).

Table 4

Experimental and Calculated Circular Dichroism ${ }^{a)}$ of Coisotectic Copolymers of Vinyl Aromatic Monomers with Optically Active (R)-3,7-dimethy1-1-octene (R37DM0)

COPOLYMER CIRCULAR DICHROISM, $\Delta \varepsilon(\lambda)$

OBSERVED REF CALCULATED REF

\begin{tabular}{|c|c|c|c|c|c|c|}
\hline $\begin{array}{l}\text { R37DMO/STYRENE } \\
(80 / 20)\end{array}$ & $-19.0(196)$ & $+10.9(188)$ & 17 & $-17.0(193)$ & $+9.6(184)$ & 24 \\
\hline $\begin{array}{l}\text { R37DMO/O-METHYLSTYRENE } \\
\quad(80 / 20)\end{array}$ & $-29.3(200)$ & $+22.3(192)$ & 17 & $-14 \cdot 3(196)$ & $+11.0(188)$ & 24 \\
\hline $\begin{array}{l}\text { R37DMO/p-METHYLSTYRENE } \\
(87 / 13)\end{array}$ & $-2.5(198)$ & $+3.2(193)$ & 19 & $-20.0(198)$ & $+21.0(188)$ & 19 \\
\hline $\begin{array}{l}\text { R37DMO / 1-VINYLNAPHTHALENE } \\
(64 / 36)\end{array}$ & $-53.5(232)$ & $+39.4(223)$ & 20 & $-95.0(228)$ & $+55.0(224)$ & 21 \\
\hline $\begin{array}{l}\text { R37DMO / 2-VINYLNAPHTHALENE } \\
(93 / 7)\end{array}$ & $+12.0(234)$ & $-10.0(232)$ & 21 & $+4.5(235)$ & $-2.5(233)$ & $2 l$ \\
\hline
\end{tabular}

a) For the Couplet in the ${ }^{1_{B}}$ electronic transition spectral region; $\Delta \varepsilon$ is given per aromatic unit and $\lambda$ in $\mathrm{nm}$. 
STYRENE/(R)-OLEFIN COPOLYMER
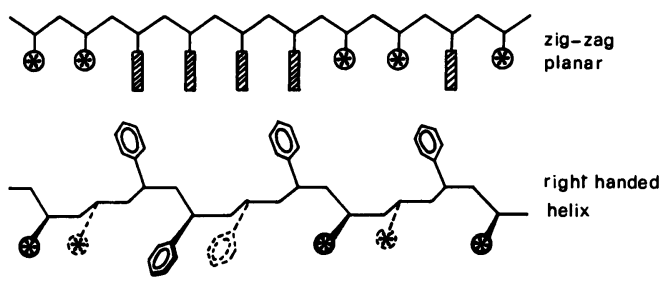

ALLYLBENZENE/(R)-OLEFIN COPOLYMER
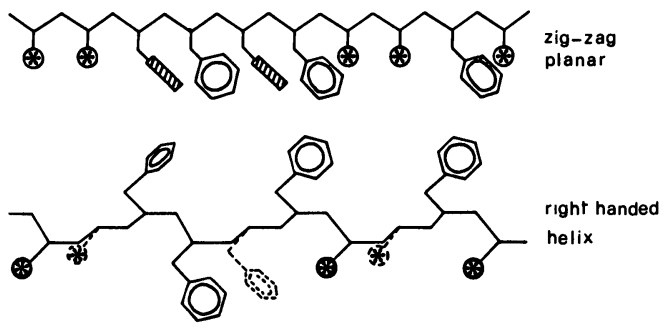
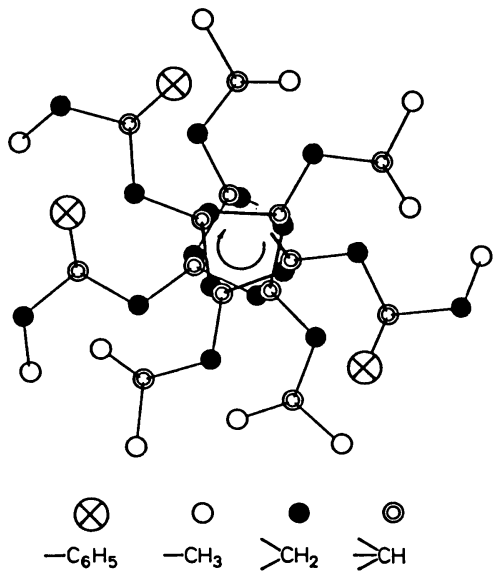

Fig. 5. End View of a Right-handed 72 Helix of the Coisotactic (R)-4-Phenyl -1-hexene/4-methy1-1-pentene Copolymer.

phenyl group (side view)
alkyl group with (R) configuration

Fig. 4. Spatial arrangement of phenyl groups in the side chains of coisotactic copolymers of styrene or allylbenzene with an optically active 1-olefin having (R)-absolute configuration.

The concept of the optically active aromatic chromophore as "conformational probe" in isotactic polymers can be furtherly extended by the use of optically active aromatic monomers. CD spectra of coisotactic copolymers of optically active 4-phenyl-1-hexene (4PH) with 4-methyl-1-pentene (4MP) allowed to correlate the sign of dichroic bands with the predominant helical screw sense (25). Indeed both the above copolymer and the isotactic homopolymer of the (R) enantiomer of 4PH showed negative dichroic bands between 270 and $250 \mathrm{~nm}$ comparable in ellipticity and with negative sign as found for previous colsotactic copolymers of vinylaromatic monomers when the right handed helicity was predominant. Moreover 4MP units give a negative contribution to the optical rotation at-Sodium D-1ine of the copolymer of about -200 per repeating unit, indicating that about $85 \%$ of these units are inserted in right-handed helices under the dissymmetric conformational effect of the $(R)$ comonomer $(12,25)$. Thus even in this case, as for the anologous copolymer of 4MP with $\mathrm{S} 4 \mathrm{MH}$, the one screw sense helical secondary structure:should-include: predominantly not only the chiral (R)-4-phenyl-1-hexene units but also the nonchiral monomer units which are than forced to assume a dissymmetric disposition as shown in figure 5 . The conclusions derived from the above results allowed to give a very reasonable explanation of the chiroptical properties of coisotactic copolymers of methacrylic esters with aromatic side chains in spite of the more complicated electronic situation due to the contemporary presence of aromatic and ester chromophore and the lower level of knowledge about the structure of these macromolecules in the crystalline state.

The most representative example of this class is offered by the copolymer of triphenylmethyl methacrylate (tritylmethacrylate, TrMA) with optically active (S) $-\alpha$-methylbenzyl methacrylate (MBMA) prepared in the presence of $\mathrm{n} . \mathrm{BuLi}$ at $-78^{\circ} \mathrm{C}$ with $\mathrm{THF}$ as the solvent. The copolymer rotatory power resulted to be dependent on composition, changing from negative to positive with increasing the content of TrMA units (26). Contemporarely an increase of the isotacticity was observed by NMR, indicating that the bulky triphenylmethyl side chains of TrMA units prevented the syndiotatctic enchainment even in THF where positive and negative ions are relatively dissociated. Copolymer chiroptical properties were thus explained by assuming that isotactic sections of TrMA units are in a single screw sense helical conformation which gives a positive contribution to optical rotation at sodium D-line. This hypothesis was confirmed by $\mathrm{CD}$ investigations showing that the sign of dichroic bands associated with both ester and aromatic chromophore change sign with composition, proving that the related electronic transitions occurr in geometrical arrangements of opposite chirality sense. An even more evident proof of the validity of this interpretation came from the ingestigation of the 
optically active homopolymers obtained by polymerization of TrMA in presence of optically active anionic catalysts. In particular the polymer obtained at $-78^{\circ} \mathrm{C}$ in toluene in the presence of (-) sparteine/Butyl lithium complex showed very high positive optical rotation at sodium $D-1$ ine and strong positive bands were also observed in the CD spectrum between 280 and $208 \mathrm{~nm}$ were the electronic transitions of the monomeric unit are located (27). The similarity of chiroptical properties of the copolymer of TrMa with the optically active comonomer SMBMA and of the homopolymer of TrMA obtained in the presence of asymmetric catalyst is a clear indication of the similar chirality in the two system, the one screw sense helicity of the isotactic chain being the most reasonable secondary structure fitting with the experimental data.

\section{NONSTEREOREGULAR POLYMERS}

Conformational analysisis of macromolecules is rendered even more complex if the chiral centers present along the backbone are distributed without following any simple rule, that is when the polymer is non stereoregular or atactic. These polymers do not crystallize and it is not possible to describe the geometrical shape of the chain in a homogeneous manner,different situations being clearly possible in sections with different microtacticity. On the other side the mentioned recent trends in polymer science oriented towards specialized applications make very useful the identification of even local order. Indeed chiroptical techniques can supply often this type of information as the optical rotatory properties and the circular dichroism of suitable chromophores are determed by short range interactions which can be specifically connected to definite local structural situations. Accordingly several examples have been reported showing that either the aromatic or the carbonyl chromophore of a nonchiral monomer in nonstereoregular random copolymers with an optically active comonomer display chiroptical properties which do not linearly depend on composition (28). This result brought to the hypothesis that in the subject copolymers the nonchiral monomer was in a chiral spatial arrangement and the resulting contribution to chiroptical properties was maximun in absolute value, for a defined sequence distribution thus proving the occurrence of specific interactions between chromophore containing units.

Such behaviour was observed for the random copolymers of N-vinylcarbazole (NVC) with (-)-menthyl-acry late or methacrylate prepared by free radical initiators which, in spite of the fairly low stereoregularity, showed dichroic bands in the absorbing region of carbazole, the corresponding molar dichroic absorption per NVC unit increasing with increasing the content of the (-)-menthyl derivative up to about $80 \%$ and then decreasing (29).

Particular mention deserve in this contest the random copolymers of p-vinyltrifluoroacetophe none (VTFA) with optically active (-) menthylacrylate prepared by free radical initiators (30). These copolymers showed between 280 and $250 \mathrm{~nm}$, where the lowest energy transition of the che toaromatic group is located, two dichroic bands of similar ellipticity and opposite sign having the typical shape of an exciton splitting cuplet due to electrostatic interactions between two or more chromophores disposed in a mutually dissymmetric spatial arrangement. For both bands the ellipticity per VTFA unit does not vary monotonically with composition reaching the highest absolute value for 30-40\% VTFA units. This behavior was assumed as a clear indication that in addition to single VTFA units, also interactions between units were contributing to $\mathrm{CD}$. In order to give this last contribution, and in particular to give rise to exciton splitting of chromophore electronic transitions, sequences of VTFA units should be in such a conformation that the side chain chromophores result disposed in relative geometrical disposition of a predominant chirality. Such a situation can be clearly regarded as a local conformational order of the chain.

Chiroptical properties which seemed to be connected with local dissymmetric conformations were also observed for random copolymers of trans-4-vinylstylbene (VS) with the same optically active comonomer (-) menthylacrylate (MtA), obtained again in the presenec of free radical initiators(31). Indeed the optical rotation at the sodium $D-1$ ine is negative for a con tent of MtA units larger than $30 \%$, whereas positive values of optical activity are observed for lower contents. The contribution of Vs units to the rotatory power, calculated by subtra cting from copolymer rotation that of the coresponding homopolymers mixture, is positive and is maximized in the range of $40-70 \%$ VS units. As for the VTFA/MtA copolymers, in this case also the $C D$ showed a couplet in the region of the lowest energy transition of trans stilbene, the ellipticity of both negative and positive band being the highest in absolute value for the 50/50 copolymer in agremet with the model assuming the occurrence of short chain sections disposedin a dissymmetric conformation of one prevailing chirality. By light irradiation in the stilbene chiromophore a trans-cis photoisomerization of the aromatic side 
chains is induced. The consequent change of shape from trans planar to cis nonplanar of the side chain is accompanied by a complete disappearance of the above CD couplet thus suggest. ing that the local conformational order is destroied by light (32). The trans to cis isomerization of the stilbene chromophore produces also a considerable decrease of polarizability and this phenomenon could at least in part be responsible for the photoinduced change of chi roptical properties.

At present it is not possible to exclude that sequences of the cromophore containning monomers in the mentioned radical copolymers have a certain degree of stereoregularity induced by the asymmetric field created by the optically active comonomer. Would this be the case, the observed $\mathrm{CD}$ couplet could probably arise from chain sections which are substantially isotactic. Calculation of CD curves and NMR investigation should allow to give a definitive answer to this question. In any case it is of interest to consider that similar copolymers of (-)MtA with styrene, vinylnaphthalene and p-acryloxybenzophenome shows no couplet and dichroic bands monotonically increasing with the content of chiral comonomer (-)MtA(28). $\mathrm{CD}$ couplet in the aromatic chromophore absorbing region were also observed in polymers without potential stereoregularity such as linear polyethylenimine provided chiral chromophoric pendants are grafted on it. Thus with optically active 2-(thymin-1-y1)-propionyl group as grafted pendant a couplet was observed in the CD associated with the base chromophores (33). In analogy to the indications deriving from theoretical studies on dinucleotides this result was taken as an indication that base stacking in these polymers has a screw sense which implies the existence of local helical structure, the role of the polyethylenimine macromolecular chain being to mantain the bases in a close vicinity.

\section{FINAL REMARKS}

The meaning and the future interest of the use of chiroptical techniques for studying conformation of macromolecules are at present broader and more stimulating with respect to the past. Indeed the original approach was to investigate structurally simple syhthetic macromolecules: to get well defined information to be used for a better understanding of the relationships between conformation and optical activity in more complex biological polymers (16). In these last analogous investigation are made more difficult by the contemporary presence of different chromophores ( 3 ) as well as tertiary and quaternary structure (2).

At present, as already mentioned in the introduction,optically active polymers are finding applications in several highly specialized areas such as chromatographic resolution of racemates (34), asymmetric catalysis (35), chiral reagents (36), synenzymes (37) and so on. Future even more sophisticated applications can be expected in biology and medicine where specific interactions and stereochemical recognition are often necessary. For all these applications a knowledge of the local secondary structure determined by nearest neighbors interections are absolutely needed and chiroptical techniques can provide the related indications.

\section{REFERENCES}

1. R.W. Woody, J. Polymer Sci., Macromol. Rev., 12, 181-321 (1977)

2. F. Ciardelli and P. Salvadori, Eds."Fundamental Aspects and Recent Developments in ORD and $C D "$, HEYDEN, London 1973

3. F. Ciardelli and O. Pieroni, Chimia, 34, 301-307 (1980)

4. G. Natta, Makromol. Chem., 35, 93-131 (1960) and references therein.

5. G. Allegra, P. Corradini and P. Ganis, Makromol. Chem., 90, 60-65 (1966)

6. P. Pino, F. Ciardelli, G.P. Lorenzi and G. Montagnoli, Makromol. Chem., 61, 207-224 (1963)

7. S. Pucci, M. Aglietto, P.L Luisi and P. Pino, in "Conformational Analysis", Academic Press Inc., 1971, 203-218

8. V. Petraccone, P. Ganis, P. Corradini and G. Montagnoli, Eur. Polymer J., 8, 99-105 (1972)

9. O. Bonsignori and G.P. Lorenzi, J. Polymer Sci., A-2, $8, \overline{1639-1649 \text { (1970) }}$

10. P. Pino, F. Ciardelli and M. Zandomeneghi, Ann. Rev. Phys. Chem., 21, 561-608 (1970) and references therein

11. O. Pieroni, F. Ciardelli and G. Stigliani, Chim. Ind., (Milan), 52, 289 (1970)

12. C. Carlini, F. Ciardelli and P. Pino, Makromol. Chem., 119, 244-248 (1968)

13. T.M. Birshtein and P.L. Luisi, Vysokomol. Soed., 6, 1238-1243 (1964)

14. C. Bertucci, D. Pini, C. Rosini and P. Salvadori, Atti VI Convegno AIM, Pisa 1983 , pp. 91-93

15. P. Neuenschwander and P. Pino, Eur. Polymer J., 19, 1075-1079 (1983) 
16. F. Ciardelli, E. Chiellini, C. Carlini, O. Pieroni, P. Salvadori and R. Menicagli, J. Polymer Sci., Polymer Symposia, 62, 143-171 (1978)

17. F. Ciardelli, P. Salvadori, C. Carlini and E. Chiellini, J. Amer. Chem. Soc., 94, 65366538 (1972)

18. P. Salvadori, L. Lardicci, R. Menicagli and C. Bertucci, J. Amer. Chem. Soc., $94,8658-$ 8600 (1972)

19. C. Righini, Thesis, University of Pisa, 1977

20. F. Ciardelli, P. Salvadori, C. Carlini, R. Menicagli and L. Lardicci, Tetrahedron Letters, (1975), 1779-1782

21. F. Ciardelli, C. Righini, M. Zandomeneghi and W. Hug, J. Phys. Chem., 81, 1948-1953 (1977)

22. E. Chiellini, R. Solaro and F. Ciardelli, Makromol. Chem., 183, 103-114 (1982)

23. C. Bertucci, C. Carlini, F. Ciardelli, C. Rosini and P. Salvadori, Polymer Bulletin, 5 , 535-542 (1981)

24. W. Hug, F. Ciardelli and I. Tinoco, Jr., J. Amer. Chem. Soc., 96, 3407-3410 (1974)

25. C. Carlini, F. Ciardelli, L. Lardicci and R. Menicagli, Nakromol. Chem., 174, 27-37 (1973)

26. Y. Okamoto, K. Suzuki and H. Yuki, J. Polymer Sci., Polymer Chem.Ed., 18, 3043-3051 (1980)

27. Y. Okamoto, K. Suzuki, K. Ohta, K. Hatada and H. Yuki, J. Amer. Chem. Soc., 101, 47634765 (1979)

28. F. Ciardelli, M. Aglietto, C. Carlini, E. Chiellini and R. Solaro, Pure\&Appl. Chem., $54,521-532$ (1982)

29. E. Chiellini, R. Solaro, G. Galli and A. Ledwith, Adv. Polymer Sci., 62, 143-169 (1984)

30. A. Altomare, C. Carlini, F. Ciardelli and E.M. Pearce, J. Polymer Sci., Polymer Chem. Ed., 21, 1693-1698 (1983)

31. A. Altomare, C. Carlini, M. Panattoni and R. Solaro, Macromolecules, 17, 2207-2212 (1984)

32. A. Altomare, C. Carlini, F. Ciardelli, M. Panattoni, R. Solaro and J.L. Houben, Macromolecules, in press (1985)

33. C.G. Overberger and Y. Morishima, J. Polymer Sci., Polymer Chem. Ed., 18 1267-1277 (1980)

34. H. Yuki, Y. Okamoto and I. Okamoto, J. Amer. Chem. Soc., 102, 6356-6358 (1980)

35. Y. Okamoto, S. Honda, I. Okamoto and H. Yuki, J. Amer. Chem. Soc., 103, 6971-6973 (1981)

36. F. Ciardelli, E. Chiellini, C. Carlini and M. Aglietto, Pure\&Appl. Chem., 52, 1857-1864 (1980) 